\title{
Stéroides sexuels et ostéoporose chez l'homme
}

\author{
Erick LEGRAND, Virginie SIMON, Maurice AUDRAN
}

Service de Rhumatologie. CHU d'Angers, 49033 ANGERS Cédex.

\section{RÉSUMÉ}

Les études épidemiologiques récentes ont confirmé que l'ostéoporose était fréquente chez l'homme. Après 50 ans, $18 \%$ des hommes seront victimes d'au moins une fracture ostéoporotique et 6 à $8 \%$ auront une fracture de l'extrémité supérieure du fémur. Dans $60 \%$ des cas environ la perte osseuse est secondaire à des facteurs étiologiques intriqués : corticothérapie prolongée, hypogonadisme franc, intoxication tabagique ou alcoolique, maladie digestive ou hépatique chronique. L'ostéoporose masculine primitive ou idiopathique, qui représente environ $40 \%$ des patients, touche souvent des hommes jeunes, entre 40 et 60 ans, indemnes de toute autre pathologie. Plusieurs voies pathogéniques sont actuellement explorés pour comprendre les raisons de cette perte osseuse chez ces patients : les facteurs génétiques, un éventuel déficit cellulaire de la formation osseuse mais surtout le rôle des stéroides sexuels biodisponibles. La carence en androgènes s'accompagne d'une accélération du remodelage osseux au profit de la résorption et d'une perte osseuse franche, prédominant dans le secteur trabéculaire. En fait l'action des androgènes sur le tissu osseux est double : directe, en raison d'un rôle stimulant de la testostérone sur la formation osseuse mais surtout indirecte par l'intermédiaire de l'estradiol, produit par aromatisation de la testostérone, qui régule le remodelage osseux et freine la résortpion osseuse. Finallement la densité osseuse et le risque de fracture sont mieux corrélés avec les taux sériques d'estradiol biodisponible et de Sex Hormone Binding Globulin (SHBG) qu'avec le taux plasmatique de testostérone, chez les hommes âgés de plus de 60 ans.

Mots clés : ostéoporose, homme, stéroides sexuels, testostérone, estradiol, physiopathologie

\section{INTRODUCTION}

Toutes les études épidémiologiques récentes montrent l'incidence élevée de l'ostéoporose chez l'homme après 50 ans et en particulier la fréquence des fractures vertébrales et la gravité des fractures de l'extrémité supérieure du fémur de l'homme âgé, dont le taux de mortalité à 1 mois est deux à trois fois supérieur à celui de la femme [49]. Que cette ostéoporose soit secondaire à un hypogonadisme, un alcoolisme, une corticothérapie prolongée ou qu'elle soit primitive (sans facteur étiologique décelable par les examens usuels), les stéroïdes sexuels occupent une place importante, mais non exclusive, dans le déterminisme de la perte osseuse et des fractures. Ce sont les études consacrées à l'ostéoporose qui ont permis de comprendre le rôle que jouent la testostérone et l'estradiol biodisponibles dans le contrôle physiologique du remodelage osseux chez l'homme.

\section{EPIDÉMIOLOGIE DES FRACTURES}

1. Les fractures de l'extrémité supérieure du fémur (ESF)

Les fractures de l'ESF représentent un problème majeur de santé publique : en 1990 on estimait à 1,7 million le nombre de ces fractures dans le monde. On admet actuellement que $25 \%$ environ des fractures de l'ESF surviennent chez l'homme [45]. A partir de 50 ans le risque de survenue d'une fracture de l'ESF, pendant le reste de la vie, est estimé entre $6 \%$ et $10 \%$ dans le sexe masculin (contre $17 \%$ pour les femmes). [19].

L'âge moyen de survenue de la fracture de l'ESF en France est de 73,1 ans dans le sexe masculin et correspond à l'âge moyen d'espérance de vie. En raison des conditions patho-

Correspondance :

Erick Legrand, Service de Rhumatologie, CHU d'Angers, 49033 ANGERS Cédex. Tel (33) 0241353576 ; Fax (33) 02 41353570 ; E-mail : erlegrand@chu-angers.fr 
logiques associées à la survenue de ces fractures, leur pronostic est médiocre : la morbidité et surtout la mortalité sont plus élevées dans le sexe masculin : le taux de mortalité du premier mois est estimé de 10 à $14 \%$ contre $5 \%$ chez la femme [31]. Toutes les projections épidémiologiques sont pessimistes : en l'absence d'interventions thérapeutiques spécifiques, l'incidence des fractures de l'ESF sera multipliée par 2 à 3 dans les 30 années à venir [29].

\section{Les autres fractures périphériques}

L'incidence des fractures de l'avant-bras est très inégalement répartie entre les deux sexes : seuls 10 à $15 \%$ de ces fractures surviennent chez l'homme. Comme chez la femme, la survenue de cette fracture est un facteur de risque important de survenue ultérieure d'une fracture de l'ESF [39].

Par contre les fractures de côtes, les fractures de l'humérus proximal et les fractures de la cheville sont fréquentes. Une étude australienne récente, portant sur une cohorte de 820 hommes de plus de 60 ans, a montré que l'incidence de ces fractures, chiffrée respectivement à 42 , 21 et 21 (pour 10000 personnes-années), était proche de l'incidence des fractures vertébrales (42 pour 10000 personnes-années) [41] .

\section{Les tassements vertébraux}

Une enquête radiographique effectuée sur l'ensemble de la population blanche de Rochester (USA) entre 1985 et 1989, a évalué l'incidence annuelle à 145 pour 100000 femmes et à 73 pour 100000 hommes [13]. En utilisant le fichier Medicare (Etats-Unis, 1986-1989) de 151986 patients de race blanche hospitalisés pour une fracture vertébrale, l'incidence est de 171 hospitalisations pour 100000 femmes et 99 pour 100000 hommes [28]. Ces deux études montrent que l'incidence des fractures vertébrales, plus élevée chez les femmes, augmente de façon exponentielle avec l'âge. Mais ce sexe ratio proche de 2 n'est pas retrouvé dans des études radiographiques récentes. Ainsi pour Davies et coll [15] la prévalence des déformations vertébrales dans une cohorte de 529 hommes du Nebraska est chiffrée à $29 \%$ à 50 ans, à $35 \%$ à 60 ans et à $40 \%$ après 80 ans contre respectivement $11 \%, 17 \%$ et $45 \%$ pour les femmes. Une étude européenne récente [43] montre également que la prévalence de ces fractures est identique dans les deux sexes. Les fractures vertébrales multiples, vraiment caractéristiques de l'ostéoporose, sont toutefois moins fréquentes chez les hommes [27].

\section{LES CONDITIONS BIOMÉCANIQUES DE SURVENUE DE LA FRACTURE CHEZ L'HOMME}

Depuis la conférence internationale de consensus de Hong Kong en 1993, l'ostéoporose est définie comme une affec- tion diffuse du squelette caractérisée par une masse osseuse basse et des altérations micro-architecturales du tissu osseux conduisant à une augmentation de la fragilité osseuse et à un risque accru de fractures .

L'existence d'une relation étroite entre masse osseuse et risque fracturaire a été démontrée par plusieurs études longitudinales et prospectives utilisant la densitométrie osseuse $[23,34,41,52]$. Des travaux récents suggèrent également que la survenue des fractures est influencée par l'anatomie des pièces osseuses (diamètre et longueur du col fémoral, épaisseur et hauteur des corps vertébraux) [54] mais également par la qualité du tissu osseux. La qualité du tissu osseux, concept composite, dépend des proportions respectives d'os cortical et d'os trabéculaire au sein d'une pièce squelettique, de la structure de l'os cortical, du degré de minéralisation du tissu osseux, de la qualité du collagène de type I, du niveau de remodelage et de la micro-architecture osseuse trabéculaire $[9,35]$ c'est à dire de l'organisation spatiale et du degré de connexion des travées osseuses.

Enfin après 70 ans la survenue de fractures, en particulier de l'ESF, est directement médiée par le risque de chute, lui même dépendant de l'état neurosensoriel et musculaire [48]

\section{FACTEURS ÉTIOLOGIQUES DE LA PERTE OSSEUSE ET DE L'OSTÉOPOROSE}

La survenue d'une ostéoporose chez un homme résulte, dans $60 \%$ des cas environ, de l'existence de facteurs étiologiques susceptibles de modifier l'acquisition du capital osseux, la cinétique de la perte osseuse, l'intégrité de la microarchitecture trabéculaire ou de majorer le risque de chute. Pour clarifier l'analyse des facteurs étiologiques, il est possible de séparer ces déterminants en 3 catégories : facteurs génétiques ou familiaux, facteurs d'environnement et de mode de vie et enfin les facteurs pathologiques, déterminants essentiels de la perte osseuse chez l'homme adulte.

\section{Facteurs génétiques et familiaux}

Il est admis que 60 à $80 \%$ de la variance du pic de masse osseuse dépend de facteurs génétiques. Deux études récentes confirment le rôle d'un faible capital osseux initial dans la génèse d'une ostéoporose chez l'homme. L'analyse d'une cohorte de 600 patients par Soroko et coll. montre que les patients qui ont un antécédent familial d'ostéoporose ont une densité osseuse plus basse sur le rachis ou sur l'ESF [57]. Cohen-Solal et coll. ont mesuré la densité osseuse des frères, des soeurs et des enfants de 38 hommes ostéoporotiques : que l'ostéoporose soit primitive ou secondaire à une intoxication alcoolo-tabagique, la densité rachidienne et fémorale est également abaissée chez les frères et soeurs [11]. Les gènes qui pourraient être en cause ne sont pas actuellement identifiés. 


\section{Facteurs d'environnement et de mode de vie}

\section{a) Activité physique}

Sous l'effet de l'entrainement physique on observe une augmentation de la masse osseuse qui intéresse l'os trabéculaire et l'os cortical [37]. Warhatig-Glynn et coll. ont ainsi montré que la densité osseuse fémorale des hommes de 65 ans dépend de leur force musculaire quadricipitale et de leurs antécédents sportifs [64]. A l'inverse, une faible activité physique exerce un effet négatif sur la densité osseuse rachidienne chez l'homme vers 50 ans [5]. Les hommes dont la force musculaire se situe dans le quartile inférieur ont ainsi une densité plus basse de $6 \%$ et ceux qui n'ont pas fait de sport au cours de l'adolescence ont une densité osseuse plus basse de 3 à $4 \%$. La force musculaire quadricipitale et le niveau d'activité physique influencent le risque de fractures rachidiennes et périphériques [41] tandis que l'inactivité multiplie le risque de fracture de l'ESF par 3 [48].

\section{b) Ration calcique et vitaminique $D$}

La carence calcique et vitaminique $D$ est un déterminant de la perte osseuse chez l'homme âgé, après 75 ans. Chez l'homme le taux de la PTH s'élève avec l'âge et les taux de la 25 hydroxyvitamine $D$ et de la 1-25 dihydroxyvitamine D diminuent au cours du vieillissement. Ces modifications biologiques, associées à la perte osseuse qui survient chez l'homme âgé, peuvent être prévenues par une supplémentation calcique et vitaminique $\mathrm{D}$.

\section{Facteurs pathologiques}

De nombreux facteurs étiologiques sont associés à la survenue d'une ostéoporose chez l'homme mais leur fréquence et leur impact pathologique réel restent encore controversés. Plusieurs auteurs $[22,30,51,53$,] ont analysé les pathologies associées à la survenue d'une ostéoporose vertébrale. Ces études descriptives suggèrent que 50 à $60 \%$ des patients ont une ostéoporose secondaire et que les pathologies les plus impliquées sont l'hypercorticisme, l'hypogonadisme, l'intoxication alcoolique ou tabagique, les maladies hépatodigestives.

\section{a) Hypercorticisme}

C'est l'une des étiologies essentielles de l'ostéoporose masculine [44]. Les corticoïdes négativent la balance calcique en réduisant l'absorption digestive du calcium et en inhibant la réabsorption tubulaire du calcium au niveau rénal. $\mathrm{Au}$ niveau du tissu osseux, ils dépriment directement l'activité des ostéoblastes et leurs capacités de synthèse protéique et favorisent leur apoptose. En revanche, la résorption osseuse est légèrement augmentée pendant les premiers mois du traitement, en raison de l'hyperparathyroïdie secondaire à la carence calcique. De plus les corticostéroïdes freinent la fonction gonadotrope chez l'homme : les effets délétères de cette carence hormonale s'additionnent à l'action directe des glucocorticoïdes sur le tissu osseux.

La densitométrie permet une évaluation de la raréfaction osseuse induite par la corticothérapie et donne des indications sur le risque fracturaire. La perte osseuse, prédominante dans le secteur trabéculaire, est rapide la première année de traitement (de l'ordre de $5 \%$ ) puis se poursuit plus lentement les années suivantes (environ $2 \%$ / an). Ce retentissement osseux, particulièrement variable d'un sujet à l'autre, est influencé par le capital osseux préalable, la nature de la maladie initiale, les doses quotidiennes ou cumulées de corticostéroïdes.

\section{b) Hypogonadisme}

L'enquête étiologique réalisée devant une ostéoporose masculine découvre un hypogonadisme chez environ 15 $\%$ des patients. La fréquence de cette pathologie est toutefois variable en fonction des séries entre 5 et $33 \%$, en raison de biais de recrutement et de variations dans la définition même de l'hypogonadisme.

Les ostéoblastes, responsables de la formation osseuse, possèdent des récepteurs aux androgènes mais aussi de très nombreux récepteurs aux oestrogènes [61]. Ces cellules sont de plus capables de convertir par aromatisation la testostérone en oestradiol. Il est donc probable que les androgènes agissent non seulement directement sur le tissu osseux mais également par l'intermédiaire des oestrogènes. Le syndrome de Klinefelter, l'hypogonadisme d'origine hypophysaire, l'hyperprolactinémie, les séquelles d'une orchite (ourlienne), l'hémochromatose génétique et les traitements par les agonistes de la GnRH [24] sont les étiologies les plus fréquentes de l'hypogonadisme masculin. Les conséquences de l'hypogonadisme sur le squelette semblent dépendre de la période de la vie ou survient cette carence en hormone mâle. Avant la puberté, l'hypogonadisme est responsable chez l'animal et chez l'homme d'un retard d'acquisition du capital osseux avec un déficit marqué sur le secteur cortical [62]. Les études animales suggèrent que le mécanisme histologique en cause est un défaut de la formation osseuse périostée avec diminution de l'épaisseur corticale [60]. Le simple retard pubertaire semble également associé avec une réduction de la densité osseuse [21]. Chez l'homme adulte, le déficit en androgènes est responsable d'une perte osseuse plus marquée dans le secteur trabéculaire. Après castration la perte osseuse lombaire est ainsi rapide (environ $-5 \%$ à $7 \% /$ an) mais mal corrélée au taux plasmatique de la testostérone [17]. L'analyse des travaux réalisés chez l'homme [24] ou chez le rat castré $[60,63,66]$ suggèrent fortement qu'une privation brutale en androgènes est suivie :

- d'une accélération du remodelage osseux au profit de la 
résorption et d'une perte rapide en secteur spongieux, proche de ce que l'on observe après la ménopause chez la femme

- d'une augmentation de la résorption endostéale et de la porosité corticale [66]

- d'une phase plus tardive de dépression de la formation osseuse [60]

- de l'installation fínale de profondes altérations de la microarchitecture osseuse trabéculaire qui annoncent la survenue des fractures spontanées [35].

La supplémentation précoce en testostérone permet d'obtenir une croissance et une maturation osseuse satisfaisante chez l'adolescent hypogonadique [65], corrige l'augmentation du remodelage osseux provoqué par la carence. Elle majore la densité osseuse, tout particulièrement durant la première année de traitement [4], chez les patients ostéopéniques, peut-être en stimulant dans certaines circonstances la formation osseuse [2]. La poursuite au long cours du traitement par voie intra musculaire mais également transcutanée [4], est efficace pour maintenir la densité osseuse dans les limites de la normale. Par contre sa prescription tardive laisserait persister un déficit du contenu minéral osseux au niveau du col fémoral [66].

\section{c) Alcoolisme}

Les effets de l'alcool sur la densité osseuse et le risque fracturaire ne sont pas univoques et dépendent du niveau de la consommation d'alcool. Une consommation modérée d'alcool (environ $150 \mathrm{gr}$ d'alcool par semaine soit environ 2 verres de vin par jour) est associée dans plusieurs études épidémiologiques récentes avec une densité osseuse plus élevée sur l'ESF [64], le rachis lombaire [5] ou le radius [20]. Le mécanisme de cet effet favorable de l'alcool sur la densité osseuse n'est pas totalement élucidé. Il pourrait résulter d'une interaction avec le système endocrinien, l'alcool favorisant la production d'androgènes par les surrénales et l'aromatisation de la testostérone en oestradiol [20].

Par contre la maladie alcoolique s'accompagne souvent d'une ostéoporose fracturaire 58]. Les études portant sur l'évolution des marqueurs du remodelage osseux après ingestion d'alcool [33, 42, 47] et les études histomorphométriques montrent que l'alcool déprime directement la fonction ostéoblastique et la formation osseuse. En revanche, ses effets sur les ostéoclastes sont discutés : la résorption est réduite dans certaines études [6] mais normale dans d'autres [10, 16, 18, 38]. L'intoxication tabagique, les carences en protéines, en calcium, en vitamine $D$ et en magnésium, l'insuffisance gonadique [8], la diminution de la masse musculaire [14] et de l'activité physique expliquent également la perte osseuse de l'alcoolique. La réversibilité potentielle de l'ostéopénie après sevrage alcoolique est suggérée par certaines études $[47,38]$ mais n'est pas actuellement tout à fait établie.

\section{d) Le tabagisme}

Le rôle du tabac dans la survenue d'une ostéoporose masculine est suspecté depuis les travaux de Seeman [53] montrant que le risque de fracture vertébrale était multiplié par 2,3 chez les grands fumeurs de plus de 60 ans, indépendemment de la consommation d'alcool. Slemenda et al. [56] ont montré que le taux de perte osseuse mesuré au radius était plus important chez les fumeurs et semblait corrélé au nombre de cigarettes. Ollenbach et al., [26] ont confirmé que la perte osseuse était mesurable sur l'ESF, corrélée au nombre de cigarettes et potentiellement réversible à l'arrêt du tabac. Il a été montré chez les femmes que la consommation de tabac était associée avec un petit poids, une diminution de l'absorption calcique et un taux plus bas d'oestrogènes. Aucune explication physiopathologique spécifique n'est par contre disponible chez l'homme.

\section{e) Les maladies hépatodigestives}

Les maladies hépatodigestives sont souvent associées à la survenue d'une ostéoporose chez l'homme [22, 53, 51]. L'analyse de ce cadre étiologique est difficile. Les pathologies impliquées sont multiples : la gastrectomie, les résections intestinales, les entérocolopathies inflammatoires, les syndromes de malabsorption, les cirrhoses alcooliques ou non, les pancréatites chroniques, les transplantations hépatiques $[12,46,50]$. Les déterminants physiopathogéniques sont nombreux et souvent associés chez un même patient : carence calcique ou vitaminique $\mathrm{D}$, carences protéiques, consommation d'alcool ou de tabac, traitement par les glucocorticoïdes, prise répétée d'antiacides (qui provoquent une hypophosphorémie).

5. Les autres étiologies sont plus rares : polyarthrite rhumatoïde, hypercalciurie, hyperthyroidie, hémochromatose, mastocytose, ostéogénèse imparfaite, homocystinurie, syndrome de Marfan, tubulopathies chroniques en particulier avec fuite phosphorée. L'ostéoporose est une des complications les plus fréquentes de la transplantation d'organe : rein, foie et cœur mais le contexte pathologique est alors évident.

\section{L'OSTÉOPOROSE MASCULINE PRIMITIVE}

L'ostéoporose masculine primitive ou idiopathique, qui représente environ $40 \%$ des patients, est une pathologie mystérieuse. Elle touche souvent des patients jeunes, entre 40 et 60 ans, indemnes de toute autre pathologie.

Les équipes de recherche travaillent actuellement dans plusieurs voies pathogéniques qui ne s'excluent pas mutuellement : 
- La piste génétique en faveur de laquelle plaide la relation étroite entre la densité osseuse des patients et celles de leurs parents (père, mère et fratrie) $[11,57]$

- La piste cellulaire en raison du fréquent déficit de la formation osseuse observé sur les analyses histomorphométriques (déficit de la fonction ostéoblastique ?)

- La piste hormonale compte tenu du rôle important que semblent jouer la testostérone biodisponible, l'estradiol biodisponible et la Sex Hormone Binding Globulin dans le contrôle du remodelage osseux chez l'homme [36].

\section{STÉROIDES SEXUELS ET OSTÉOPOROSE PRIMITIVE}

Nous savons depuis longtemps que l'hypogonadisme est une étiologie possible de l'ostéoporose chez l'homme. La carence en androgènes s'accompagne d'une accélération du remodelage osseux au profit de la résorption et d'une perte osseuse franche, prédominant dans le secteur trabéculaire. Mais il est établi qu'une fraction de la testostérone peut être convertie en estradiol par l'intermédiaire d'une aromatase. Les ostéoblastes, cellules clés dans le contrôle du remodelage osseux, possèdent l'aromatase, le récepteur aux androgènes et le récepteur aux oestrogènes.

De nombreux arguments laissent penser que l'action des androgènes sur le tissu osseux est donc double :

- directe d'une part en raison d'un rôle stimulant de la testostérone sur la formation osseuse

- indirecte par l'intermédiaire de l'estradiol, produit par conversion de la testostérone, sous l'influence de l'aromatase.

Plusieurs arguments laissent penser que cette action indirecte, par l'intermédiaire de l'estradiol, est la plus importante :

La densité osseuse des hommes âgés, au-delà de 65 ans est mieux corrélée avec les taux sériques d'estradiol et de Sex Hormone Binding Globulin (SHBG) qu'avec le taux plasmatique de testostérone. Greendale et coll. [25], Khosla et coll. [32] puis Szulc et coll. [59] ont confirmé que le taux sérique de l'estradiol bio-disponible, c'est à dire non lié à la SHBG, était un facteur prédictif de la densité osseuse chez l'homme. Dans une étude longitudinale récente BarretConnor et coll. [3] ont montré que les hommes dont les taux d'estradiol bio-disponible étaient les plus bas avaient également un risque plus élevé de fracture vertébrale.

Cette diminution des taux d'estradiol biodisponible pourrait être la conséquence

- d'un déficit de production de la testostérone (substrat de l'aromatase)

- mais également d'une élévation anormale de la SHBG.
Cette glycoprotéine de 373 acides aminés capable de lier, avec une forte affinité, l'estradiol et la testostérone, assure le transport de ces stéroïdes sexuels et régule leur activité. En raison d'une vitesse de dissociation trop lente, les stéroïdes liés à la SHBG ne sont pas actifs dans les tissus cibles $[40,55]$. Dans une étude transversale récente, nous avons montré chez 80 patients et 40 contrôles (âge moyen 49,7 ans) souffrant d'une ostéoporose que le taux de la SHBG était anormalement élevé chez plus de deux tiers d'entre eux, corrélé à la densité osseuse fémorale $(\mathrm{r}=0,38)$, corrélé aux marqueurs biochimiques de la résorption osseuse et surtout associé au risque de tassement vertébral, toute majoration d'un écart-type de la SHBG multipliant le risque de fracture par deux [36]. Center et coll., [7] ont également montré chez 437 hommes âgés que le risque de fracture était multiplié par 1,7 pour toute augmentation d'un écarttype de la SHBG, même après ajustement pour l'âge, le poids, la force musculaire quadricipitale et la densité osseuse fémorale.

Quelle est la place de la testostérone dans le traitement de l'ostéoporose primitive en l'absence d'hypogonadisme ?

L'utilisation de la testostérone par voie IM a été évaluée par Anderson et coll. [1] dans une étude ouverte, sans groupe contrôle, en administrant tous les 15 jours $250 \mathrm{mg}$ d'esters de testostérone à 21 patients ostéoporotiques mais eugonadiques. Les résultats montrent un gain de densité osseuse lombaire de $5 \%$ sur 6 mois associé à une réduction significative des marqueurs biochimiques de la résorption osseuse, sans effets secondaires importants cardiovasculaires. L'influence du traitement sur le risque de fracture n'est pas actuellement connu.

\section{CONCLUSIONS}

Il est maintenant acquis que les stéroïdes sexuels et en particulier la testostérone et l'estradiol biodisponibles participent largement au contrôle du remodelage osseux, qui conditionne lui même l'acquisition du capital osseux au moment de la puberté et le maintien de la masse osseuse et de la qualité osseuse à l'âge adulte.

De multiples circonstances pathologiques peuvent s'accompagner d'un hypogonadisme et d'une carence en testostérone plus ou moins profonde. Si le syndrome de Klinefelter, l'hypogonadisme d'origine hypophysaire, l'hyperprolactinémie, les séquelles d'une orchite, l'hémochromatose génétique et les traitements par les agonistes de la GnRH sont des étiologies classiques, la corticothérapie prolongée, l'alcoolisme chronique et le vieillissement provoquent aussi des modifications hormonales qui diminuent la formation osseuse, accélèrent la résorption osseuse et la perte osseuse et bouleversent la microarchitecture du réseau trabéculaire osseux. La 
survenue de fractures spontanées, caractéristiques de l'ostéoporose, est alors inéluctable en l'absence de thérapeutiques adaptées.

La supplémentation hormonale par la testostérone est efficace et indispensable devant une ostéoporose associée à un hypogonadisme franc. L'intérêt de la même thérapeutique au cours de l'ostéoporose primitive, en particulier chez le sujet âgé, demande encore à être validé par des études complémentaires.

\section{REFERENCES}

1. ANDERSON F.H., FRANCIS R.M., PEASTON R.T., WASTELL H.J. : Androgen supplementation in eugonadal men with osteoporosis : effects of six months treatment on markers of bone formation and resorption. J. Bone Miner. Res., 1997, 12 : $472-478$.

2. BARAN D.T., BERGFELD M.A., TEITELBAUM S.L., AVIOLI LV. : Effects of testosterone therapy on bone formation in an osteoporotic hypogonadal male. Calcif. Tiss. Res., 1978, 97, 19-30.

3. BARRET-CONNOR E, MUELLER J.E, VON MÜHLEN D.G, LAUGHLIN G.A., SCHNEIDER D.L., SARTORIS D.J. : Low levels of estradiol are associated with vertebral fractures in older men but not women: the Rancho Bernardo Study. J. Clin. Endocrinol. Metab., 2000; 85: 219-223.

4. BEHRE H.M., KLIESCH S., LEIFKE E., LINK T.M. and NIESCHLAG E. : Long term effect of testosterone therapy on bone mineral density in hypogonadal men. J. Clin. Endocrinol. Metab., 1997, 82, 2386-2390.

5. BENDAVID E.J., SHAN J., BARRET-CONNOR E. : Factors associated with bone mineral density in middle-aged men. $\mathrm{J}$. Bone Miner. Res., 1996, 11 : 1185-1190.

6. BIKLE D.D., GENANT H.K., CANN C., RECKER R.R., HALLORAN B.P., STREWLER J. : Bone disease in alcohol abuse. Ann. Intern. Med., 1985, 103, 42-48.

7. CENTER J.R., NGUYEN T.V., SAMBROOK P.N., EISMAN J.A. : Hormonal and biochemical parameters and osteoporotic fractures in men. J. Bone Miner. Res., 2000, 15: 1405-1411.

8. CHAPPARD D., ALEXANDRE C., RIFFAT G. : L'os du cirrhotique : relation entre la masse osseuse et la testostéronémie plasmatique. Nouv. Press. Med., 1983, 12, 524

9. CHAPPARD D., LEGRAND E., BASLE M.F., FROMONT P., RACINEUX J.L., REBEL A., AUDRAN M. : Altered trabecular architecture induced by corticosteroids : a bone histomorphometric study. J. Bone Miner Res., 1996, 11 : 676-685.

10. CHAPPARD D., PLANTARD B., PETITJEAN M., ALEXANDRE C., RIFFAT G. : Alcoholic cirrhosis and osteoporosis in men light and scanning electron microscopic study. J. Stud. Alcohol., 1991, 52, 269-274.

11. COHEN-SOLAL M.E., BAUDOIN C., OMOURI M., KUNTZ D., DE VERNEJOUL M.C. : Bone mass in middle-aged osteoporotic men and their relatives: familial effect. J. Bone Miner. Res., 1998, $13: 1909-1914$.
12. COMPSTON J.E., HORTON L.W.L., AYERS A.B., TIGHE J.R., CREAMER B. : Osteomalacia after small-intestinal resection . Lancet, 1978, 1, 9-12.

13. COOPER C., ATKINSON E.J., O'FALLON W.M., MELTON III LJ. : Incidence of clinically diagnosed vertebral fractures : a population based study in Rochester, Minnesota, 1985-1989. J. Bone Miner. Res., 1992, 7 : 221-227.

14. CRILLY R.G., DELAQUERRIERE-RICHARDSON L. : Current bone mass and body weight changes in alcoholic males. Calcif. Tissue Int., 1990, 46, 169-172.

15. DAVIES K.M., STEGMAN M.R., HEANEY R.P., RECKER R.R. : Prevalence and severity of vertebral fracture : the saunders county bone quality study. Osteoporos is Int., 1996, 6 : 160-165.

16. DE VERNEJOUL M.C., BIELAKOFF J., HERVE M., GUERIS J., HOTT M., MODROWSKID,KUNTZ D. : Evidence for defective osteoblastic function. A role for alcohol and tobacco consumption in osteoporosis in middle aged men. Clin. Orthop., $1983,179,107-115$.

17. DEVOGELAER J.P., DE COOMAN S., NAGANT DE DEUXCHAISNES C. : Low bone mass in hypogonadal males : effect of testosterone substitution therapy, a densitometric study. Maturitas, 1992, 15, 17-23.

18. DIAMOND T., STIEL D., LUNZER M., WILKINSON M., POEN S. : Ethanol reduces bone formation and may cause osteoporosis. Ann. Intern. Med., 1989, 86, 282-288.

19. ELFFORS I., ALLANDER E., KANIS J.A. et al., : The variable incidence of hip fractures in Southern Europe : the MEDOS Study. Osteoporosis Int., 1994, 4: 253-263.

20. FELSON D.T., ZHANG Y., HANNAN M.T., KANNEL W.B., KIEL D.P.. : Alcohol intake and bone mineral density in elderly men and women. Am. J. Epidemiol., 1995; 42 : 485-492.

21. FINKELSTEIN J.S., NEER R.M., BILLER B.M.K., CRAWFORD J.D., KLIBANSKI A.: Osteopenia in men with history of delayed puberty. N. Engl. J. Med., 1992, 326, 600-604.

22. FRANCIS R.M., PEACOCK M, MARSHALL D.H., HORSMAN A., AARON J.E. : Spinal osteoporosis in men. Bone Miner., 1989, 5: 347-358.

23. GÄRDSELL P., JOHNELL O., NILSSON B.E. : The predictive value of forearm bone mineral content measurements in men. Bone 1990, $11: 229-232$.

24. GOLDRAY D., WEISMAN Y., JACCARD N., MERDLER C., CHEN J., MATSKIN H.: Decreased bone density in elderly men treated with the gonadotropin-releasing hormone agonist decapeptyl. J. Clin. Endocrinol. Metab., 1993, 76, 288-290.

25. GREENDALE G.A., EDELSTEIN S., BARRET-CONNOR E. : Endogenous sex steroid and bone mineral density in older women and men: the Rancho Bernardo Study. J. Bone Miner. Res., 1997, 12: 1833-1843.

26. HOLLENBACH K.A., BARRET-CONNOR E., EDELSTEIN S.L., HOLBROOK T.L. : Cigarette smoking and bone mineral density in older men and women. Am. J. Public. Health., 1993, 83, 1265-1270.

27. ISMAIL A.A., O'NEILL T.W., COOPER C, SILMAN A.J. : The European Vertebral Osteoporosis Study Group. Risk Factors for vertebral deformity in men : relationships to number of vertebral deformities. J. Bone Miner. Res., 2000, 15 : 278-283. 
28. JACOBSEN S.J., COOPER C., GOOTLIED M.S., GOLDBERG J., YAHNKE D.P., MELTON L.J. : Hospitalization with vertebral fracture among the aged : a national populationbased study 1986-1989. Epidemiology, 1992, 3 : 515-518.

29. KANNUS P., PALVANEN M., NIEMI S., PARKKARI J., JÄRVINEN M. : Epidemiolgy of osteoporotic pelvic fractures in elderly people in Finland : sharp increase in 1970-1997 and alarming projections for the new millennium. Osteoporosis Int., $2000 ; 11: 443-448$.

30. KELEPOURIS N., HARPER K.D., GANNON F., KAPLAN F.S., HADDAD J.G. : Severe osteoporosis in men. Ann. Intern. Med., 1995, 123:452-460.

31. KELLIE S.E., BRODY J.A. : Sex-specific and race-specific hip fracture rates. Am. J. Public. Health., 1990, 80 : 326-328.

32. KHOSLA S., MELTON J., ATKINSON E.J., O'FALLON W.M., KLEE G.G., RIGGS B.L. : Relationship of serum sex steroid levels and bone turnover markers with bone mineral density in men and women: a key role for bioavailable estrogen. J. Clin. Endocrinol. Metab., 1998, 83: 2266-2274

33. LAITIMEN K., LAMBERGALLARDT C., TUNNINEN K., KAROMEN S.L., YLIKAHNI R., VALIMAKI M. : Effects of 3 weeks moderate alcohol intake on bone and mineral metabolism in normal men. Bone Miner. 1991, 13, 139-152.

34. LEGRAND E., CHAPPARD D., PASCARETTI C. et al. : Bone mineral density and vertebral fractures in men. Osteoporos is Int., $1999,10: 265-270$.

35. LEGRAND E., CHAPPARD D., PASCARETTI C., DUQUENNE M,KREBS S., ROHMER V., BASLE M.F, AUDRAN M. : Trabecular bone microarchitecture, bone mineral density and vertebral fractures in male osteoporosis. J. Bone Miner. Res., 2000, 15: 13-19.

36. LEGRAND E., HEDDE C., GALLOIS Y., MATHIEU E., BOUX DE CASSON F., CHAPPARD D., BASLE M.F., AUDRAN M. : The sex binding protein in male osteoporosis. Bone 2001, $29: 90-95$.

37. LEGRAND E., LE DANTEC P., MEADEB J., PERDRIGER A., CHALES G., PAWLOTSKYY. : Les effets réels du sport sur la masse osseuse de la femme. Rev. Rhum., 1992, 59 : 335-339.

38. LINDHOLM J., STEINICHE T., RASMUSSEN E., THAMSBORG G., NIELSEN I.O., BROKSTEDTRASMUSSEN H., STORM T., HYLDSTRUP L., SCHOU C. : Bone disorder in men with chronic alcoholism : a reversible disease ? J. Clin. Endocrinol. Metab., 1991, 73, 118-124.

39. MALLMIN H., LJUNGHALLS, PERSSON I., NAESSEN T., KRUSEMO U.B., BERGSTROM R. : Fracture of the distal forearm as a forecaster of subsequent hip fracture : a population based cohort study with 24 years follow-up. Calcif. Tissue Int., 1993, 52 : 269-272.

40. MERCIER C., ALFSEN A., BAULIEU E.E. : A testosterone binding globulin. In: Proceedings of the second symposium on the steroid hormones. Ghent ed. Excerta Medica International Congress Series 1966,

41. NGUYEN T.V., EISMAN J.A., KELLY P.J., SAMBROOK P.N. : Risk factors for osteoporotic fractures in elderly men. Am. J. Epidemiol., 1996, 144, 255-263.
42. NIELSEN H.K., LUNDBY, RASMUSSEN K., CHARLES P., HANSEN C. : Alcohol decreases serum osteocalcin in a dose dependant way in normal subjects. Calcif.Tissue Int., 1990, 46, 173-178.

43. O'NEILL T.W., FELSENBERG D., VARLOW J., COOPER C., KANIS J.A., SILMAN A.J. : The Osteoporosis Study Group. The prevalence of vertebral deformity in european European Vertebral men and women : the european vertebral osteoporosis study. J. Bone Miner. Res., 1996, 11 : 1010-1018.

44. ORCEL P. : Ostéoporose cortisonique : nouvelles approches. Rev. Rhum., 1997, 64 : 717-723.

45. ORWOLL E.S., KLEIN R.F. : Osteoporosis in men. Endocrine Review, 1995, $16: 87-116$.

46. PARFITT A.M., MILLER M.J., FRAME B., VILLANUEVA A.R., RAO D.S., OLIVER I., THOMSON D.L. : Metabolic bone disease after intestinal bypass for treatement of obesity. Ann. Inter. Med., 1978, 89, 193-199.

47. PEPERSACKT T., FUSS M., OTERO J., BERGMANN P., VALSAMIS J., CORVILAIN J. : Longitudinal study of bone metabolism after ethanol withdrawal in alcoholic patients. J. Bone Miner. Res., 1992, 7, 383-387.

48. POOR G., ATKINSON E.J., LEWALLEN D.G., O'FALLON W.M., MELTON L.J. III. : Predictors of hip fractures in elderly men. J. Bone Miner Res., 1995, 10 : 1900-1907.

49. POOR G., ATKINSON E.J., LEWALLEN D.G., O'FALLON W.M., MELTON L.J. III. : Age-related hip fractures in men : clinical spectrum and short term outcomes. Osteoporosis Int., 1995, 5: 419-426.

50. RAO D.S., HONASOGE M.: Metabolic bone disease in gastrointestinal, hepatobiliary and pancreatic disorders. In Favus MJ (ed). Primer on the bone diseases and disorders of mineral metabolism. Third edition. ASBMR. Lipincott-Raven. 1996, pages 306-311.

51. RINGE J.D., DORST A. : Osteoporosis in males: pathogenesis and clinical classification. Proceedings on the Fourth International Symposium on Osteoporosis and Consensus Development Conference. Hong Kong. 1993, C Christiansen and B Riis, editors. p 184-186.

52. ROSS P.D., KIM S., WASNICH R.D. : Bone density predicts fracture risk in both men and women. J. Bone Miner. Res., 1996, 11 : Suppl. 1, S127.

53. SEEMAN E., MELTON L.J., O'FALLON W.M., RIGGS B.L. : Risk factors for spinal osteoporosis in men. Am. J. Med., 1983, 75 : 977-983.

54. SEEMAN E. : Advances in the study of osteoporosis in men. In Papapoulos SE, Lips P, Pols HAP, Johnston CC, Delmas PD editors. Osteoporosis ' 96 . Proceedings of the 1996 World Congress on Osteoporosis. Elsevier Science. The Netherlands, 1996, 341358.

55. SELBY C. : Sex hormone binding globulin: origin, function and clinical significance. A.Clin. Biochem., 1990, 27 : 532-541.

56. SLEMENDA C.W., CHRISTIAN J.C., REED T., REISTER T.K., WILLIAMS C.J., JOHNSTON C.C. : Long term bone loss in men : effects of genetic and environmental factors. Ann. Intern. Med., 1992, $117: 286-291$. 
57. SOROKO S.B., BARRET-CONNOR E., EDELSTEIN S.L., KRITZ-SILVERSTEIN D. : Family history of osteoporosis and bone mineral density at the axial skeleton : the Rancho Bernardo Study. J. Bone Miner Res., 1994, 9: 761-769.

58. SPENCER H., RUBIO N, RUBIO E. : Chronic alcoholism. Frequently overlooked cause of osteoporosis in men. Am. J. Med., 1986, 80, 393-397.

59. SZULC P., MUNOZ F., CLAUSTRAT B., GARNERO P., MARCHAND F., DUBOEUF F., DELMAS P.D. : Bioavailable estradiol may be an important determinant of osteoporosis in men: the MINOS study. J. Clin. Endocrinol. Metab., 2001, 86: 192-199.

60. TURNER R.T., HAMMNON K.S. : Differential effects of gonadal function in bone histomorphometry in male and female rats. J. Bone Miner. Res., 1989, 4, 557-563.

61. VANDERSCHUEREN D., BOUILLON R. Androgens and bone. Calcif. Tissue Int., 1995, 56, 341-346.

62. VANDERSCHUEREN D., VAN HERCK E., SUIKER A.M., VISSER W.J., SCHOT L.P., CHUNG K., LUCAS R.S,, EINHORN T.A., BOUILLON R. : Bone and mineral metabolism in the androgen-resistant (testicular feminized) male rat. J. Bone Miner Res., 1993, 8, 801-809.

63. VERHAS M., SCHOUTENS A. :The effect of orchidectomy on bone metabolism in aging rats. Calcif. Tissue Int., 1986, 39, 7477.

64. WARHAFTIG GLYNN N.W., MEILAHN E.N., CHARRON M., ANDERSON S.J., KULLER L.H., CAULEY J.A. : Determinants of bone mineral density in older men. J. Bone Miner Res., 1995, 10, 1769-1777.

65. WEBSTER B., HODGKIN W. : Influence of androgen therapy on growth rate of hypogonadal adolescent boys. Proc. Soc. Exp. Med., 1940, 45, 72-75.

66. WINK C.S, FELTS W.J.L. : Effects of castration on the bone structure of male rats : a model of osteoporosis. Calcif Tissue Int., 1980, 32, 77-82.

67 .WONG F.G.H., PUN K.K, WANG C. : Loss of bone mass in patients with Klinefelter's syndrome despite sufficient testosterone replacement. Osteoporosis Int., 1993, 3, 3-7.

\section{ABSTRACT}

Sex steroids and osteoporosis in men Erick LEGRAND, Virginie SIMON, Maurice AUDRAN

Several epidemiological studies have shown that about 25 per cent of hip fractures and 20 per cent of symptomatic vertebral fractures occur in men. The lifetime risk of hip fracture was estimated to be about 6 to 8 per cent and the risk of any osteoporotic fracture was estimated to be about 18 per cent in 50 -year-old white men. In about $60 \%$ of cases in men, bone loss is secondary to several pathological conditions, such as long-term steroid therapy, severe hypogonadism, smoking or alcohol abuse or gastrointestinal disorders. In $\mathbf{4 0 \%}$ of cases, osteoporosis is primary or idiopathic in men between the ages of $\mathbf{4 0}$ and $\mathbf{6 0}$ years. Genetic factors, a defect of boneforming cells or abnormal serum levels of bioavailable sex steroids could explain bone loss and fragility fractures in these men. It has been shown that hypogonadism is associated with a marked increase in bone remodelling and particularly in bone resorption with a dramatic loss in trabecular bone. It is now known that testosterone is partly transformed into estradiol by aromatase. Testosterone may therefore act on bone in two ways: it directly stimulates bone formation and estradiol regulates bone remodelling and inhibits bone resorption. Finally, in men over the age of 60 without hypogonadism, it has been shown that bone mineral density and fracture risk were better correlated with serum levels of bioavailable estradiol and Sex Hormone Binding Globulin than with serum testosterone levels.

Key-words: osteoporosis, men, sex steroids, testosterone, estradiol, pathophysiology 